\title{
VULNERABILIDAD Y ACTIVOS FAMILIARES FRENTE A RIESGOS. Caso de ESTUdio EN IXIL, YuCATÁN
}

\author{
Vulnerability and Household Assets against Risks. Case Study in Ixil, Yucatan \\ Norma Cecilia Sandoval-Ayala \\ Denise Soares-Moraes
}

Resumen: En esta contribución se presenta un estudio de caso sobre percepciones sociales respecto a la vulnerabilidad de los activos familiares frente a riesgos de desastres en el municipio de Ixil, estado de Yucatán, México. Se aplicaron 81 encuestas a representantes de hogares de la comunidad y se realizaron diecinueve entrevistas semiestructuradas. Se encontró que los huracanes son un factor clave tanto en la percepción sobre la vulnerabilidad, como en las situaciones de riesgo que ocasionan pérdidas de activos en la región.

Palabras clave: vulnerabilidad, activos familiares, riesgos, amenazas y huracanes.

Abstract: In this paper we present a case study in the town of Ixil, Yucatan State, Mexico, about the vulnerability of household assets against disaster risk. We applied 81 surveys to local people and realized interviews with nineteen people. We found that hurricanes are a key factor in both the perception of vulnerability and risk situations that cause loss of assets in this area.

Keywords: vulnerability, household assets, risks, threats, hurricanes.

\footnotetext{
Norma Cecilia Sandoval Ayala, maestra en gobiernos y políticas públicas por la Universidad Autónoma de Yucatán, México. Temas de especialización: vulnerabilidad, gestión de riesgos y evaluación de políticas públicas. Correo electrónico: ncecisandoval@hotmail.com.

Denise Soares Moraes, doctora en antropología social por la Universidad Nacional Autónoma de México. Investigadora del Instituto Mexicano de Tecnología del Agua. Temas de especialización: vulnerabilidad, equidad de género, gestión de riesgos y metodologías participativas. Correo electrónico: denisefsoares@yahoo.com.mx.
}

Enviado a dictamen: 22 de mayo de 2014.

Aprobación: 15 de agosto de 2014.

Revisiones: 1 . 


\section{Introducción}

$\amalg$ estado de Yucatán es una zona especialmente amenazada por los efectos del cambio climático. Una variación de pocos grados en la temperatura superficial de los océanos tendrá impactos amplios y complejos, como la posible elevación del nivel del mar, cambios en los regímenes de precipitación pluvial y en las capacidades del suelo para asimilar el exceso de humedad, el incremento e intensidad de ciclones tropicales y huracanes o cambios en las características ambientales de los ecosistemas mismos (IPCC, 2007; Botello y Villanueva-Fregoso, 2010; Yáñez-Arancibia y Day, 2010). Los fenómenos asociados al cambio climático ponen en riesgo no sólo los ecosistemas, sino además la seguridad de los recursos y activos de las poblaciones que habitan en las zonas costeras de la Península de Yucatán.

El cambio climático supone un factor clave al amplificar las interacciones entre riesgo por desastre y pobreza porque, por un lado, aumentan las amenazas hidrometeorológicas y, por otro, dicho cambio incide en el declive de los ecosistemas de los que dependen las actividades económicas con las que se sustenta la población. Por tal motivo, los impactos del cambio climático en Yucatán implican un enorme desafío para quienes elaboran las políticas públicas si consideramos que, en función de la geografía de la península, la exposición de la población a la variación climática es muy alta y pone en riesgo los esfuerzos para reducir la pobreza y promover la justicia social.

Las comunidades pobres de los municipios costeros de Yucatán conviven con tres factores subyacentes de riesgo de pérdidas por desastre: bajos ingresos y medios de vida vulnerables, ecosistemas amenazados y niveles muy bajos de protección social. A menos que se afronten estos factores, las personas pobres continuarán sufriendo pérdidas desproporcionadas por desastres (EIRD-ONU, 2009), independientemente de lo que pudiera sumarse por los impactos asociados con el cambio climático.

A pesar de la existencia de estudios físicos y geográficos sobre los impactos del cambio climático, que han permitido establecer escenarios a nivel territorial, ${ }^{2}$ a la fecha no se cuenta con suficiente información a escala reducida sobre sus efectos económicos y sociales $y$, para entenderlos desde el contexto local, es necesario combinar el conocimiento de los pobladores de las comunidades con la información científica sobre el clima (CARE, 2010).

De acuerdo con Olivé: "Para todo tipo de riesgo que afecte intereses colectivos de un sector de la sociedad o de la naturaleza, es éticamente indispensable la participación pública en el proceso que va de la identificación a la gestión del riesgo" (2004: 291). Además de la importancia ética de la participación pública en los análisis de gestión del riesgo por amenazas climáticas, los procesos participativos permiten a las personas conocer y entender mejor los riesgos climáticos a los que se exponen, y sus ventajas y oportunidades para afrontarlos en tanto se recupera el conocimiento local con autoridad creíble para informar e influir en la política (Chambers, 2010).

Tomando como premisa que la vulnerabilidad al cambio climático puede variar entre regiones, comunidades, hogares e individuos, y además en función de elementos como el sexo, la edad, la clase social, la etnia o la religión, es necesario realizar diagnósticos de vulnerabilidad social de las comunidades costeras de la península que consideren las capacidades sociales, institucionales e individuales que permitan planificar con efectividad acciones de adaptación, a la vez que se recupera la información científica del cambio climático, pero también las percepciones de los propios pobladores.

¿Cuáles son las principales situaciones de riesgo que ocasionaron pérdidas de activos entre las familias de Ixil? ¿Qué desastre tuvo mayor peso dentro de la trayectoria de vulnerabilidad socioeconómica de las familias? ¿Cuál es la relación entre las amenazas y la economía local? ¿Cuáles son las estrategias familiares empleadas ante los diferentes tipos de riesgos? ¿Cómo la gente percibe su vulnerabilidad ante distintos tipos de riesgo? Con el fin de hacer frente a estas lagunas se elabora la presente contribución, que tiene como objetivo mejorar la comprensión acerca 
de cómo las familias del municipio de Ixil perciben sus vulnerabilidades, así como identificar acciones de adaptación a favor de la población.

\section{Un acercamiento a la vulnerabilidad social basada en movilización de activos}

En la década de los noventa, los estudiosos del impacto del cambio climático comúnmente utilizaron un enfoque que permitió la modelación de escenarios para la elaboración de mapas de riesgo sobre el cambio climático. En estos mapas, la vulnerabilidad generalmente se construyó sobre índices o indicadores relativos de pobreza o de desarrollo humano; sin embargo, fue necesario que la cartografía de la vulnerabilidad permitiera ir más allá del análisis de la exposición a los riesgos para incorporar factores como la susceptibilidad y la capacidad de adaptación. Para ello, había que explicar, entonces, por qué independientemente del tipo de amenaza o de la fuente de la amenaza, algunos individuos, grupos o comunidades tenían una mayor o menor capacidad para prepararse, responder y recuperarse de los distintos riesgos (Blaikie et al., 1994). Se llegó a la conclusión de que, ante cualquier tipo de amenaza, había una condición preexistente de cierta independencia del riesgo (Cutter et al., 2009).

La necesidad de emprender nuevos estudios que permitieran cerrar las brechas en la investigación sobre cambio climático y reducción de la pobreza haciendo énfasis en el desarrollo de capacidades de adaptación, dio origen a la utilización del enfoque de vulnerabilidad social basado en activos ${ }^{3}$ (asset vulnerability qpproach). Este enfoque toma en cuenta la vulnerabilidad social, pero también las capacidades construidas en función de la disponibilidad de activos o capitales, y la forma en que los individuos, familias o comunidades transforman sus recursos en activos, así como su poder para expandir su base de activos a través de la participación con otros actores (Sen, 1981; Chambers, 1989; Moser 1998), en el entendido de que hay una fuerte interacción entre los activos de los hogares y sus fuentes de producción, que se rigen por la lógica del mercado, el Estado y la sociedad (Katzman y Filgueira, 1999).

El enfoque de vulnerabilidad social basado en activos toma en cuenta también las capacidades construidas en función del acceso a los diferentes tipos de recursos, activos o capitales, y centra su atención en cómo los individuos, familias, grupos sociales o comunidades movilizan sus recursos ante situaciones de riesgo. Desde este punto de vista, los activos son un portafolio de recursos - materiales y no materiales- que los pobres pueden movilizar a través de sus capacidades y estrategias de afrontamiento (coping strategies) y que pueden variar por región, comunidad, grupo social, tipo de hogar, género, edad, estación del año y período de tiempo (Chambers, 1989; Moser, 1998). En algunos marcos analíticos centrados en los medios de vida, los activos son la suma de recursos tangibles, es decir, recursos y bienes acumulados, como alimentos, dinero, ahorros, joyas, ganado, maquinaria y herramientas agrícolas, y recursos intangibles relacionados con el acceso a las oportunidades que pueden tomar la forma de apoyo o derecho de acceso ante familiares, vecinos, grupos sociales, autoridades u ONG (Chambers y Conway, 1992).

Los marcos analíticos de capitales de la comunidad (community capitalsframework), que surgen de los modelos del construccionismo social, han definido los capitales de la comunidad como aquellos que representan activos en todos los aspectos de la vida comunitaria. Un activo se convierte en capital cuando se invierte en la comunidad (Flora et al., 2004). Los activos o capitales más utilizados en los enfoques relacionados con la vulnerabilidad social son los capitales humanos, físicos, sociales, culturales, políticos, financieros y naturales, cuya definición general, así como su contribución a la vulnerabilidad social, establecemos en la tabla 1.

Es clave comprender la relación entre los diferentes capitales o activos para determinar los puntos de inflexión que pueden llevar hacia un círculo vicioso o virtuoso, el cual conducirá a la adopción de medidas concretas de adaptación y mejora del bienestar de las unidades domésticaslocales. Conociendolospuntos débilesdecada capital y cómo pueden afectar a los demás, se determinan 
los puntos de control que deben ser monitoreados y potenciados, así como las acciones adecuadas que se deben emprender a fin de reducir la vulnerabilidad de dicho capital y crear sinergias con otros. Por ejemplo, los procesos de evaluación participativa pueden aumentar la comprensión que las personas tengan acerca de su vulnerabilidad y el riesgo de desastres - capital humano-, situación que contribuye, a su vez, a generar iniciativas de articulación y organización social para hacer frente a los desastres — capital social—, además de favorecer un fortalecimiento del nivel de preparación institucional ante los desastres que conduzca a una respuesta más efectiva — capital político-. Ello puede redundar en menores pérdidas de infraestructura —capital físico-y de recursos naturales — capital natural-, al reducir las inversiones que se tendrán que efectuar para salir de la situación de desastre — capital financiero-.

Bebbington (2005) anota una crítica importante para los enfoques basados en activos y capitales, sobre todo en aquellos que ponen énfasis en la carencia de recursos de los hogares pobres, en los que queda difuminada la dimensión de agencia (agency). Por otra parte, en los estudios de la literatura anglófona se pone mayor atención en lo que los pobres tienen y en su capacidad para la acción. Por ello, Bebbington recomienda adoptar una perspectiva de equilibrio entre ambas posiciones, de modo que tan importante es que se valoren los recursos que tienen los individuos, hogares o comunidades, como los que no tienen, así como el margen real de agencia que las estructuras hacen posible para que las personas fortalezcan sus activos.

La vulnerabilidad social, desde la perspectiva de los activos o no, necesita siempre un punto de referencia, por ejemplo, un determinado tipo de riesgo o amenaza — la vulnerabilidad ante qué-. Un riesgo es la probabilidad de incurrir en daños o la probabilidad del daño o pérdida resultante de la situación de peligro. Por ejemplo, el inicio de la temporada de huracanes en el Atlántico incrementa la amenaza de que un huracán impacte sobre el territorio de la Península de Yucatán porque simplemente hay una mayor probabilidad de ocurrencia del evento por las condiciones favorables para el desarrollo de huracanes. Por otra parte, los desastres son eventos singulares de mayor escala que superan la capacidad local para responder de manera eficaz de modo que los damnificados se recuperen de un evento. Tanto las amenazas como los desastres se deben a muchas causas, pero normalmente se considera que éstas últimas se encuentran en la interacción entre la sociedad y los sistemas naturales - huracanes, por ejemplo-, la sociedad y la tecnología — por ejemplo, derrames de sustancias químicas - o bien dentro de la propia sociedad (Cutter et al., 2009).

Asimismo, además de la situación de riesgo debe tomarse en cuenta el contexto social específico que transforma un riesgo o amenaza en un proceso de pérdida —vulnerabilidad a-, así como las características de las unidades de análisis: individuo, hogar o grupo social —vulnerabilidad de quién-. No sólo es importante la exposición de las unidades de análisis, sino también las capacidades de cada unidad para afrontar y adaptarse ante una amenaza determinada. Esto se debe a que las personas no son sujetos pasivos, sino que cuentan con cualidades dinámicas, como su nivel de conciencia, percepciones, motivaciones, actitudes y conocimiento acerca de las amenazas —incluidas las amenazas naturales-.

$\mathrm{Y}$, por último, sumado al riesgo y a la escala, el análisis de la vulnerabilidad social requiere considerar dimensiones de tiempo, dado que se trata de estados temporales y acumulables de vulnerabilidad - ex-ante y ex-post-, como condición interna y en función de la secuencia de aparición de las amenazas climáticas.

Una vez realizado este breve recorrido por el enfoque de vulnerabilidad social basada en activos, retomamos elementos de esas propuestas conceptuales para conformar nuestro propio acercamiento al tema. Para nosotros, la vulnerabilidad supone la incapacidad de determinados grupos sociales para hacer frente, resistir y recuperarse de eventos extremos debido a la debilidad de los activos con los cuales cuentan, y por ello es un elemento clave en la ecuación del desastre. Se puede afirmar que existe una relación directamente proporcional, es decir, a mayor vulnerabilidad de los grupos sociales, mayor será el desastre resultado de 
la amenaza. La vulnerabilidad es multidimensional en tanto que involucra componentes socioeconómicos, ambientales y físicos relacionados con los activos o capitales.

\section{Contexto y método}

Ixil es la localidad cabecera del municipio del mismo nombre. Se ubica aproximadamente a unos 28 kilómetros de la ciudad de Mérida y a escasos 25 kilómetros de la costa (Orilla, 1998: 17). A pesar de que el municipio cuenta con litoral, por razones geográficas y políticas su población no tiene acceso a la explotación de los recursos del mar. Cuenta con 3728 habitantes, de los que 1903 son hombres y 1825 son mujeres, de modo que la relación hombres-mujeres es de 104.27. De acuerdo con el Catálogo de localidades indígenas ${ }^{4}$ de la Comisión Nacional para el Desarrollo de los Pueblos Indígenas (CDI, 2010), Ixil se encuentra clasificada como una localidad de interés debido a que su población indígena, predominantemente maya, ${ }^{5}$ alcanza el $39.2 \%$ de la población total.

Esta localidad se encuentra ubicada en la antigua zona henequenera del norte del estado de Yucatán y es un perfecto ejemplo de la complejidad en cuanto a diferencias entre lo urbano y lo rural. Su realidad socioeconómica, histórica y cultural se impone ante el simple criterio cuantitativo de que 2500 habitantes delimitan la frontera entre lo urbano y lo rural. Por tal motivo, Ixil es una de las localidades clasificadas por el INEGI como rural ampliada. ${ }^{6}$

De acuerdo con esta misma institución (INEGI, 2009), el municipio de Ixil se ubica entre los paralelos $21^{\circ} 08^{\prime}$ y $21^{\circ} 20^{\prime}$ de latitud norte, y los meridianos $89^{\circ} 24^{\prime}$ y 89 32'. Colinda al norte con el Golfo de México y con los municipios de Progreso, al poniente; Chicxulub Pueblo, al surponiente; Mocochá, al sur; Motul, al suroriente, y al oriente con Dzemul y Telchac Puerto. Tiene una altitud sobre el nivel del mar de entre seis y diez metros. Ocupa el $0.37 \%$ de la superficie del estado de Yucatán: 134.13 kilómetros cuadrados. El tipo de clima es semiseco muy cálido y cálido (56.71\%), seco muy cálido y cálido (40.33\%) y cálido subhúmedo, con lluvias en verano $(2.96 \%)$.

El municipio forma parte de la región noroeste del estado y se caracteriza por su alto nivel de desarrollo económico y su alta concentración de población. Sin embargo, de acuerdo con la SEDUMA (2010), ninguna de las dos condiciones se distribuye homogéneamente en la región, que se caracteriza por su gran dependencia económica con respecto al municipio de Mérida, además de por sus grandes desigualdades en cuanto a desarrollo.

De acuerdo con la clasificación del CONAPO (2010), Ixil es una localidad con un nivel de marginación alto debido principalmente a sus condiciones de pobreza y al tamaño de su población. Esto último puede significar que los pobladores enfrenten algunas carencias por falta de acceso a la educación o a algunos servicios públicos, por viviendas inadecuadas o por ingresos insuficientes. Por otro lado, conforme a la medición municipal de la pobreza efectuada por el CONEVAL (2010), el 62.3\% de la población del municipio de Ixil sufre algún tipo de pobreza: 1968 habitantes (50.4\%) viven en una situación de pobreza moderada y 466 pobladores (11.9\%) se encuentran en situación de pobreza extrema. Con relación al bienestar económico, el 67.1\% de la población vive con un ingreso inferior a la línea de bienestar, y un $20.2 \%$ de la población lo hace con un ingreso inferior a la línea de bienestar mínimo. Únicamente el 6.1\% de la población del municipio, es decir, 237 habitantes, es considerado por el CONEVAL como no pobre y no vulnerable.

En cuanto a los indicadores de privación social, el $89.2 \%$ de los pobladores observa al menos una carencia social y el $40 \%$ tiene al menos tres carencias sociales. Las carencias más observables en el municipio se deben al no acceso a la seguridad social (62.4\%), falta de acceso a servicios básicos en la vivienda (57.4\%), rezago educativo (29.8\%) y carencias por dificultades de acceso a la alimentación (28.6\%) (CONEVAL, 2010).

Desde el referente metodológico, la selección de Ixil como lugar de estudio obedeció a los siguientes criterios: a) de acuerdo con el INEGI (2011), la localidad tiene un grado de marginación alto; b) Ixil se encuentra clasificada por la CDI como una localidad de interés ${ }^{7}$ 
debido a que su población indígena alcanza el 39.2\% de la población total; c) aunque se considera dentro de las localidades de ámbito urbano, los habitantes de Ixil enfrentan algunas carencias por falta de acceso a la educación, por viviendas inadecuadas, por ingresos monetarios insuficientes y por algunas otras formas estructurales y funcionales de exclusión relacionadas con la residencia en localidades pequeñas (CONAPO, 2010); y d) los medios de sustento de una porción importante de su población dependen en su mayoría de ingresos estacionales derivados del cultivo de las hortalizas o de otras formas de empleo temporal o de ocupaciones basadas en el autoempleo.

Se aplicaron encuestas y entrevistas a actores sociales de Ixil. Para la aplicación de las encuestas se consideraron como universo de estudio 903 hogares de la localidad, y para determinar el número de encuestas por aplicar se utilizó la fórmula para la determinación de la muestra cuando el universo es finito, conforme se especifica en la tabla 2. Para la aplicación de los cuestionarios familiares, se empleó un mapa de la localidad de Ixil y, con base en el número total de manzanas, se determinó el número de cuestionarios que debía aplicarse por manzana. Los temas abordados en la encuesta fueron los siguientes: situaciones de riesgo que ocasionan pérdidas de activos, pérdidas de activos y estrategias familiares ante riesgos y percepciones sobre la vulnerabilidad ante diferentes tipos de riesgos.

Las entrevistas semiestructuradas fueron aplicadas a diecinueve informantes clave, nueve mujeres y diez hombres, pertenecientes a diferentes familias residentes en distintos barrios de la localidad. La entrevista como método cualitativo permite cruzar la información obtenida de manera cuantitativa y arrojar mayores elementos de análisis.

\section{Percepciones sobre riesgos y afectaciones a las familias}

Se comparten las percepciones de los pobladores de Ixil respecto a los diferentes tipos de riesgo que sus familias han experimentado y las consecuencias de dichas situaciones en sus activos familiares. Asimismo, se recuperan algunas historias familiares, a través de las entrevistas, que permiten apreciar los significados sociales de las distintas situaciones de riesgo.

Como se puede observar en el gráfico 1 , de todas las situaciones de riesgo que ocasionaron una crisis o calamidad económica a nivel familiar, un $27.2 \%$ se relaciona con huracanes y un $6.1 \%$ con alguna inundación o sequía. Es decir, una tercera parte (33.3\%) de las crisis económicas familiares tuvieron un origen o causa en el medioambiente. Por lo tanto, este tipo de riesgos es aún superior al de los ocasionados por la pérdida del empleo o quiebra del negocio, elementos que fueron mencionados en veintiséis ocasiones; es decir, un $22.8 \%$. En este punto, es importante señalar que, al aplicar la encuesta, nos aseguramos de que al hacer esta pregunta quedara claro, a los hombres o mujeres entrevistados, que se trataba de situaciones de riesgo que ocasionaron algún tipo de crisis económica para la familia en los últimos cinco años.

Isidoro fue el huracán que ocasionó más daños catastróficos en la localidad. Ocurrió en el año 2002; es decir, más de diez años atrás, en lugar de los cinco señalados durante la aplicación de la encuesta. A pesar de ello, el huracán Isidoro continuaba siendo, según la percepción de los habitantes de la localidad, la situación de riesgo o calamidad económica con mayor peso dentro de la trayectoria de vulnerabilidad socioeconómica de las familias. Retomamos algunos testimonios al respecto:

Cuando fue Isidoro perdimos todo, la cosecha. La casa se inundó. Luego vino el programa de empleo temporal para los dos. Tuvimos que trabajar los dos y aun así tardamos como dos años para estabilizarnos un poco (ama de casa, 36 años)

La situación económica nunca ha sido buena. Hace como trece años me casé y tuve a mis dos hijas. No podíamos mantenerlas y ahora sólo la mayor vive conmigo. La otra ha sido muy enfermiza y siempre ha vivido con sus abuelos. En ese entonces vivíamos en casa de los suegros, en Chicxulub [pueblo], y cuando fue el huracán Isidoro se llevó la casa y tuvimos que 
irnos a vivir a una casa prestada. Poco después, a él lo detuvieron por robo de autos y ahora está en la cárcel. Hace como cuatro años, me junté con otra pareja, el papá de los dos niños, pero nos separamos. Todavía no sé si fue malo o fue bueno. Ahora vivo en el pueblo, en una casa rentada, y unos amigos de mi papá me regalaron una lavadora de medio uso y la gente me lleva su ropa para lavar. Acabo de meter los papeles para Oportunidades, a ver si me llega la beca para mi hija (jefa de hogar dedicada a lavar ajeno, 28 años).

Primero fue Isidoro, que acabó con todas nuestras cosechas. Luego, hace como cuatro años, se perdió la cosecha por sequía. Tuvimos que empeñar las alhajas, y las autoridades locales apoyaron con mil pesos para tratar de recuperar la cosecha. Dos años después, nuevamente hubo pérdida de cosechas por sequía y ya no hubo apoyo de las autoridades. Finalmente tuvimos que vender nuestro auto para poder comprar semillas. Si no, ¿cómo? (horticultor, 51 años).

Para enfrentar las distintas situaciones de riesgo, las familias deben movilizar sus activos y elaborar estrategias, sean reactivas o proactivas, para superar los impactos que las crisis económicas puedan implicar para la pérdida del bienestar a nivel familiar. Sin embargo, a pesar de las estrategias que fueron llevadas a cabo por las familias para recuperarse de las crisis ante los riesgos identificados por ellos mismos, éstos ocasionaron pérdidas significativas de activos familiares.

En la tabla 3 se observan las crisis declaradas por las familias en relación con los riesgos más comúnmente identificados por los pobladores, las pérdidas de activos que ocasionaron y las diferentes estrategias que las familias llevaron a cabo para recuperarse de dichas situaciones.

Con independencia de la movilización de recursos y activos que las familias deban efectuar para enfrentar una situación de riesgo, así como de sus capacidades, dichas situaciones resultan estresantes debido a la sensación de fragilidad o vulnerabilidad para hacer frente a los choques externos. De acuerdo con la pregunta de cómo se sintieron los miembros del hogar ante las situaciones de riesgo anteriormente descritas, las respuestas para las calamidades económicas de mayor frecuencia fueron las que se evidencian en el gráfico 2, en el que se puede observar que continuaban siendo los huracanes los que, además de tratarse de los riesgos que con más frecuencia ocasionaban afectaciones económicas en los hogares, eran también los fenómenos ante los cuales la población se sentía más frágil o vulnerable (33\%).

Por otra parte, las enfermedades en el hogar, los accidentes o la muerte de algún familiar parecían inducir una mayor sensación de alta vulnerabilidad en las familias más que la pérdida del empleo o del negocio.

La alta sensación de vulnerabilidad de la población ante huracanes puede deberse a lo catastróficos que suelen ser los daños que ocasionan, en términos de bienestar económico y de pérdida combinada de varios de los activos del portafolios de las familias. Un huracán puede significar que a causa de un solo evento las familias vean amenazadas sus cosechas, viviendas, ingresos, terrenos, ganado e insumos. El período de recuperación del bienestar económico puede prolongarse hasta años, según los testimonios de algunas de las familias entrevistadas.

En este breve recorrido por las percepciones sociales sobre la vulnerabilidad y la pérdida de activos frente a riesgos, quedó patente que los huracanes constituían la variable más reconocida a nivel local como potencial fuente de desastres para Ixil. Si bien los huracanes son una fuerte amenaza exógena, no habría que menospreciar la más importante amenaza que vive la comunidad, que es endógena y estructural y que, además, interactúa con todos los otros riesgos, los potencia e impide una capacidad adecuada de respuesta y recuperación por parte de la población. Nos estamos refiriendo a la pobreza.

Las poblaciones en situación de pobreza viven de manera persistente situaciones de exclusión social, lo que plantea un conjunto de limitaciones o desventajas que las personas encuentran para acceder a los activos. Dicha situación interfiere negativamente en sus capacidades y oportunidades para responder y recuperarse frente a desastres. Según Moser (1998), 
las posibilidades de elección del modo de vida y la capacidad para superar situaciones adversas de una persona se definen de acuerdo con la estructura de activos que ésta posee, dado que dichos activos sirven para enfrentar los riesgos (sensitivity) o, en su defecto, para adaptarse a sus consecuencias (resilience). La autora concluye aseverando que ciertos individuos o familias pobres son más vulnerables que otros en función de la disponibilidad y de la movilización que éstos hacen de los distintos activos a los cuales acceden. La pobreza, por definición, limita el acceso a los activos.

\section{Conclusiones}

En esta contribución se realizó un primer acercamiento al contexto inmediato de la vulnerabilidad social de la localidad de Ixil ante los riesgos que ocasionan pérdidas de activos. Pudimos también recuperar un componente clave de la construcción social del riesgo: la percepción sobre la vulnerabilidad frente a diferentes categorías de riesgos.

Se encontró que los huracanes son un factor clave, tanto en la percepción sobre la vulnerabilidad como en las situaciones de riesgo que ocasionan pérdidas de activos. Mediante el análisis de los impactos que causaron situaciones de riesgo y crisis económicas familiares durante los últimos años, se encontró que una tercera parte de las calamidades económicas tuvieron un origen en el medioambiente y que eran los huracanes los que ocasionaban más alta sensación de vulnerabilidad en los pobladores de Ixil. En términos de impactos negativos en las trayectorias de bienestar familiares, los huracanes han representado pérdidas de activos como cosechas, viviendas, ingresos, terrenos, ganado o insumos. El período de recuperación del bienestar económico familiar era largo y demandaba una combinación de múltiples estrategias familiares de afrontamiento y adaptación.

El potencial del enfoque de vulnerabilidad social que ocasiona pérdidas de activos en situaciones de riesgo puede constituir una herramienta valiosa para generar políticas y programas que asuman el compromiso de disminuir las situaciones sociales contingentes que generan condiciones de riesgo, y promover el fortalecimiento y la diversificación de activos. Asimismo, puede potenciar las capacidades de los individuos, de los hogares y de las comunidades para que hagan frente por sí mismos a situaciones de riesgo a través del análisis de las causas subyacentes a la vulnerabilidad.

\section{Notas}

${ }^{1}$ Para efectos de esta investigación, el cambio climático es entendido como todo cambio del clima a lo largo del tiempo, tanto si es debido a la variabilidad natural como si es consecuencia de la actividad humana (IPCC, 2007).

${ }^{2}$ En la entidad se cuenta con un "Atlas de escenarios de cambio climático", documento conformado por una serie de proyecciones hacia 2020, basadas en estadísticas y cambios en el medioambiente que se han registrado en las últimas décadas y sobre los posibles impactos que podrían sufrir los sistemas naturales frente a este fenómeno (Orellana et al., 2009).

${ }^{3}$ El enfoque de vulnerabilidad y activos tiene su origen en la década de los noventa y deriva de los debates sobre desarrollo y vulnerabilidad. Se encuentra sustentado en la teoría de dotación de derechos (entitlements) y capacidades de los pobres propuesta por Amartya Sen (1981); las aportaciones presentadas por Coleman (1988) y Putman (1993) para la formulación del concepto de capital social, el modelo de acceso a los recursos (Blaikie et al., 1994), así como por aquellos enfoques que sostienen que la pobreza no sólo se relaciona con una falta de ingresos o consumo, sino también con una falta de activos (Chambers, 1989).

${ }^{4}$ El Catalogo de localidades indígenas elaborado de manera conjunta entre el INEGI y la CDI permite trascender el simple criterio lingüístico utilizado por el INEGI. La metodología de la CDI agrega como componente a la población de hogares indígenas, entendidos como aquellos donde el jefe, cónyuge o un ascendiente - madre, padre, suegra, suegrodeclaró hablar alguna lengua indígena. 
${ }^{5}$ En Yucatán, la inmensa mayoría de los indígenas se adscribe a un solo grupo étnico: los mayahablantes que representan el 99.6\% de la población indígena (Ruz, 2006).

6 El INEGI (2005) utiliza el concepto ampliado de población rural para aquellas localidades de menos de 5000 habitantes, y utiliza fuentes de información o criterios adicionales al corte por tamaño de localidad, como equipamiento urbano, cobertura de agua potable y electricidad, telefonía, servicios educativos y de salud o gubernamentales, medios de comunicación, mercados, centrales camioneras, cines, parques y plazas públicas, clubes sociales y deportivos, etcétera.

${ }^{7}$ Una localidad con menos del $40 \%$ de población indígena y más de 150 es considerada por la CDI como localidad de interés. Fuente: Catálogo de Localidades Indígenas 2010, elaborado con datos derivados del Censo de Población y Vivienda de 2010 y resultado de los trabajos entre el INEGI y metodología formulada por la CDI para la identificación y cuantificación de la población indígena de México.

\section{Referencias bibliográficas}

Bebbington, A. (1999), "Capitals and Capabilities: A Framework for Analyzing Peasant Viability, Rural Livelihoods and Poverty". En World Development, vol. 27, núm. 12, pp. 2021-2044.

Bebbington, A. (2005), "Estrategias de vida y estrategias de intervención: el capital social y los programas de superación de la pobreza”. En Arriagada, I. (ed.) Aprender de la experiencia. El capital social en la superación de la pobreza. Santiago de Chile, CEPAL/Cooperazione Italiana, pp. 21-46.

Blaikie, P., I. Cannon, I. Davis y B. Wisner, B. (1994), At Risk: Natural Hazards, People's Vulnerability, and Disasters. Londres, Routledge.

Botello, A.V. y S. Villanueva-Fragoso (2010), "Introducción”. En Botello, A.V., S. Villanueva-Fragoso, J. Gutiérrez y J.L. Rojas Galaviz (eds.), Vulnerabilidad de las zonas costeras mexicanas ante el cambio climático. Campeche, Gobierno del Estado de Tabasco/SEMARNAT-INE/UNAMICMYL/Universidad Autónoma de Campeche, pp. 1-14.
CARE (2010), Manual para el análisis de capacidad y vulnerabilidad climática (CVCA). Perú, CARE Perú. Disponible en http:/www.careclimatechange.org/ files/adaptation/CARE_CVCA_Handbook-2009Spanish.pdf [consultado el 23 de julio de 2012].

CDI (2010), Catálogo de localidades indígenas 2010. Base censo INEGI 2010 y metodología CDI. México, CDI.

Chambers, R. (2010), "Prefacio". En Manual para el análisis de capacidad y vulnerabilidad climática (CVCA). Perú, CARE Perú. Disponible en http://www. careclimatechange.org/files/adaptation/CARE_ CVCA_Handbook-2009-Spanish.pdf [consultado el 23 de julio de 2012]

Chambers, R. (1989), "Vulnerability: How the Poor Cope". En R. Chambers (ed.), Vulnerability, Coping and Policy. IDS Bulletin, vol. 37.4, Anthology 2006. Brighton, Universidad de Sussex.

Chambers, R. y G. Conway (1992), Sustainable Rural Livelihoods: Practical Concepts for the 2lth Century. Brighton, Institute of Development Studies.

Coleman, J.S. (1988), "Social Capital in the Creation of Human Capital”. En The American Journal of Sociology, vol. 94, "Supplement: Organizations and Institutions: Sociological and Economic Approaches to the Analysis of Social Structure", S95-S120.

CONAPO (2010), Índice de marginación por localidad 2010. México, CONAPO.

CONEVAL (2010), Medición de la pobreza 2010, Indicadores de pobreza, por municipio. México, CONEVAL.

Cutter, S.L. (1996), "Vulnerability to Environmental Hazards". En Progress in Human Geography, núm. 20(4), pp. 529-539.

Cutter, S.L., C.T. Emrich, J.J. Webb y D. Morath (2009), Social Vulnerability to Climate Variability Hazards: A Review of the Literature. Final Report to Oxfam America. Columbia, University of South.

De Haan, L. y A. Zoomers (2005), "Exploring the Frontier of Livelihood Research". En Development and Change, núm. 36(1), pp. 27-47.

Filgueira, C. (1998), "Welfare and Citizenship: New and Old Vulnerabilities". En O'Donnell, Guillermo y Victor E. Tokman, Poverty and Inequality in Latin America. París, University of Notre Dame Press. 
Flora, C., J. Flora y S. Fey (2004), Rural Communities: Legacy and Change. Coulder, Columbia: Westview Prees.

Foust Rodríguez, D. (2009), Capital social: una espada de dos filos. Estudio sobre capital social en Atemajac de Brizuela, Jalisco. Guadalajara, Jalisco, Universidad de Guadalajara, Centro Universitario de Ciencias Sociales y Humanidades.

García Acosta, V. (2005), "El riesgo como construcción social y la construcción social de riesgos". En Desacatos, núm. 19, pp. 11-24.

García Acosta, V. (2009), Prevención de desastres, estrategias adaptativas y capital social. En Koff, H. (ed.), Social Cohesion in Europe and the Americas: Power, Time and Space. Bruselas: P.I.E. Peter Lang, S.A. Éditions Scientifiques Internationals, pp. 115-130.

INEGI (2005), Población rural y ampliada en México. México, INEGI.

INEGI (2010), Catálogo de claves de entidades federativas, municipios y localidades. México, SEDESOL, Unidad de Microrregiones. Disponible en http://www. microrregiones.gob.mx/catloc/LocdeMun.aspx? buscar=1\&tipo=nombre\&campo=loc\&valor=ixil [consultado el 20 de julio de 2012].

INEGI (2011), Censo de población y vivienda 2010. México, INEGI.

Katzman, R. y C.H. Filgueira (1999), "Introducción”. En Katzman, R. (coord.), Activosyestructuradeoportunidades: estudio sobre las raíces de la vulnerabilidad social en Uruguay. Montevideo, PNUD/CEPAL, pp. 37-164.

Moser, C. (1998), "The Asset Vulnerability Framework. Reassessing Urban Poverty Reduction Strategies". En World Development, núm. 26(1), pp. 1-9.

Olivé, L. (2004), "Riesgo, ética y participación pública". En Luján, J.L. y J. Echeverría, Gobernar los riesgos: ciencia y valores en la sociedad del riesgo. Madrid, OEI/Biblioteca Nueva, pp. 289-309.
Orellana, R., C. Espadas, C. Conde y C. Gay (2009), Atlas. Escenarios de cambio climático en la Península de Yucatán. Mérida, Yucatán, Centro de Investigación Científica de Yucatán, A.C.

Orilla Canché, M. (1998), Monografía de Ixil: tierra de las cebollitas. Mérida, Yucatán, PACMYC/Gobierno del Estado de Yucatán/Instituto de Cultura de Yucatán, Dirección General de Culturas Populares.

Putnam, R. (1993), Making Democracy Work: Civic Traditions in Modern Italy. Princeton, Princeton University Press.

Ruz, M.H. (2006), Mayas: primera parte. México, CDIPNUD.

SEDUMA (2010), Carta urbana síntesis. Ixil. Mérida, Secretaría de Desarrollo Urbano y Medio Ambiente del Gobierno del Estado de Yucatán. Disponible en http://www.seduma.yucatan.gob. $\mathrm{mx} /$ desarrollourbano/documentos/CartasUrbanas/ Noroeste/Ixil.pdf [consultado el 25 de julio de 2012].

Sen, A. (1981), Poverty and Famines: An Essay on Entitlement and Deprivation. Nueva York, The Clarendon Press, Oxford University Press.

Wilches-Chaux, G. (1993), "La vulnerabilidad global". En Maskrey A. (comp.), Los desastres no son naturales. Bogotá, LA RED/Tercer Mundo Editores, pp. 9-50.

Woolcock, M. y D. Narayan (2000). "Social Capital: Implications for Development Theory, Research and Policy". En The World Bank Research Observer, vol. 15, num. 2, pp. 225-249.

Yañez-Arancibia, A. y J.W. Day (2010), "La zona costera frente al cambio climático: vulnerabilidad de un sistema biocomplejo e implicaciones en el manejo costero". En Rivera-Arriaga, E. et al. (eds.), Cambio climático en México. Un enfoque costero-marino, Campeche. Campeche, Universidad Autónoma de Campeche CETYS/Universidad, Gobierno del Estado de Campeche, pp. 3-22. 
Gráfico 1. Porcentaje de situaciones de riesgo que ocasionaron pérdidas de activos a las familias encuestadas

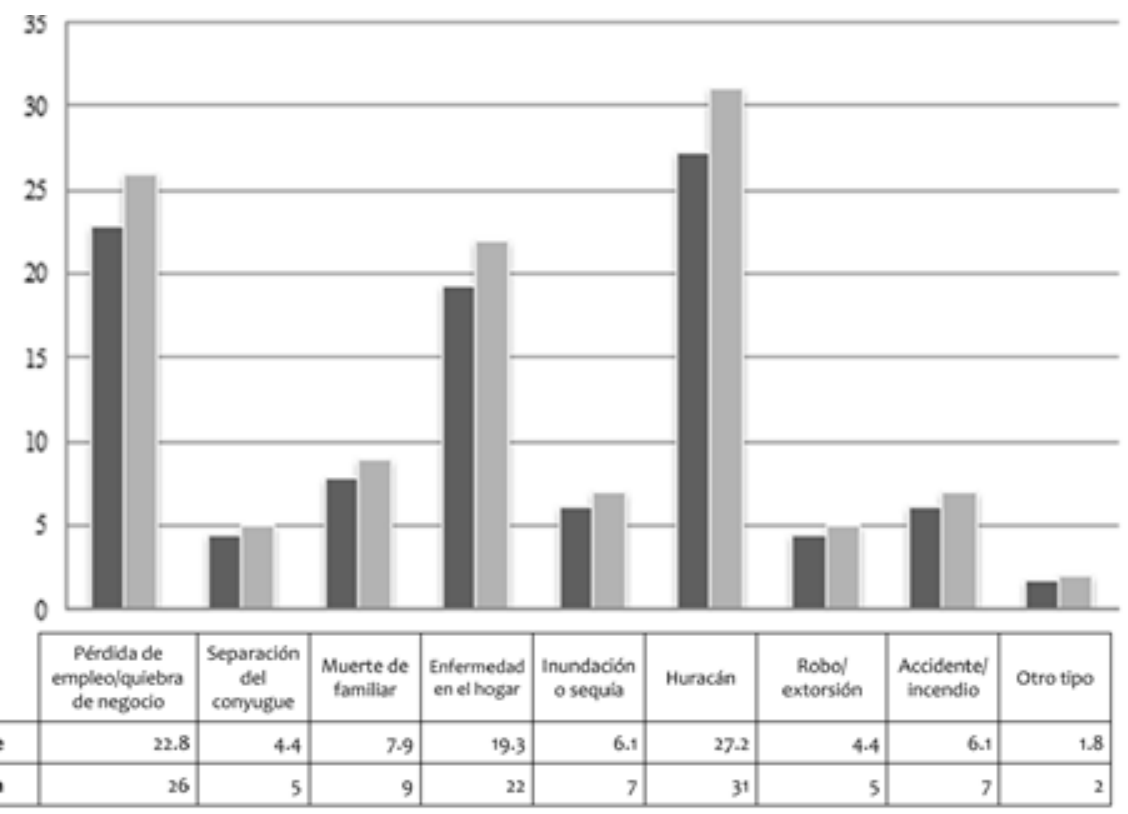

Fuente: elaboración propia.

Gráfico 2. Percepciones sobre el sentimiento de vulnerabilidad ante distintos tipos de riesgo

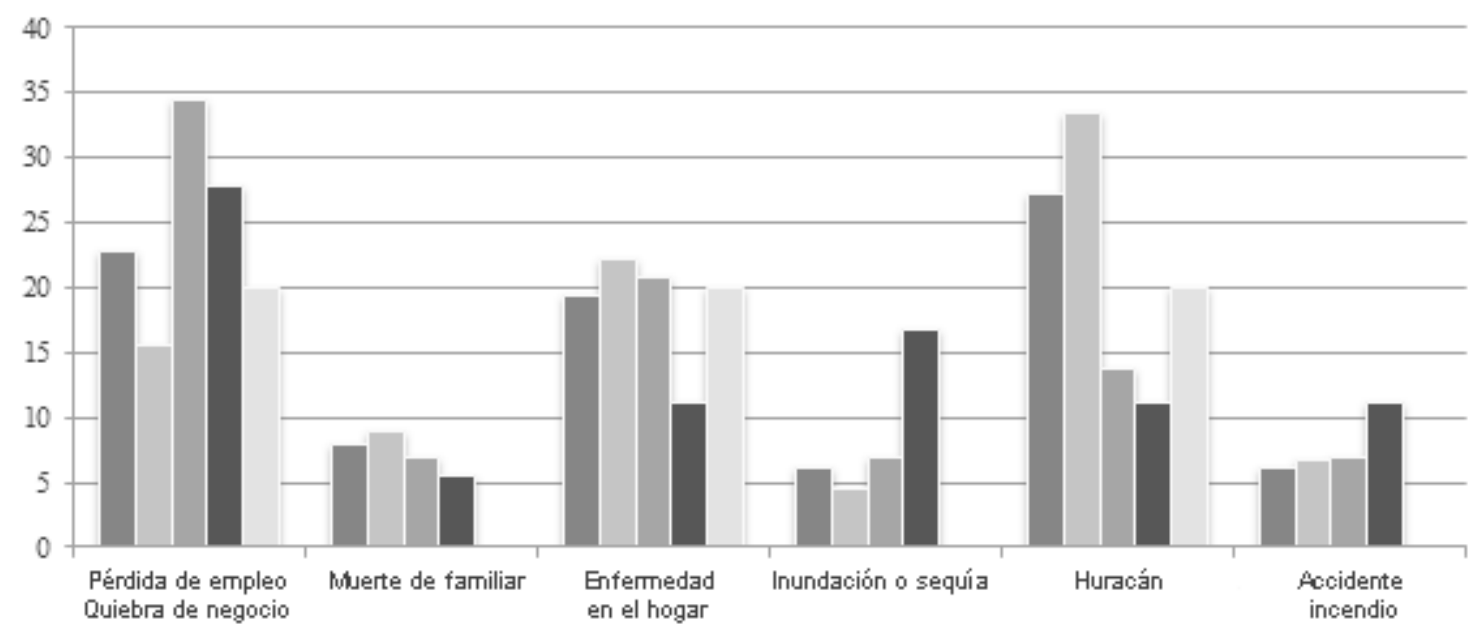

- Frecuencia =Bastante vulnerables = Wulnerables - Poco vulnerables Nada vulnerables

Fuente: elaboración propia. 
Tabla 1. Definiciones de los diferentes tipos de activos o capitales y su contribución a la disminución de la vulnerabilidad social

\begin{tabular}{|c|c|c|}
\hline Capital & Definición & $\begin{array}{l}\text { Contribución a la disminución de } \\
\text { vulnerabilidad }\end{array}$ \\
\hline Humano & $\begin{array}{l}\text { El capital humano se refiere a los recursos de que } \\
\text { disponen las personas y los hogares en términos de } \\
\text { educación, capacitación, habilidades, salud y otras } \\
\text { (adaptado de Moser, 1998). }\end{array}$ & $\begin{array}{l}\text { El buen estado de salud de las personas, } \\
\text { la educación, capacitación y habilidades } \\
\text { que determinan el retorno de su principal } \\
\text { activo, el trabajo (Moser, 1998). }\end{array}$ \\
\hline Social & $\begin{array}{l}\text { Bajo ciertas condiciones es un bien público (Foust, } \\
\text { 2009) que incluye relaciones, lazos de confianza, } \\
\text { de reciprocidad o redes que pueden ser utilizados } \\
\text { para soportar estrategias de sobrevivencia, de } \\
\text { acumulación (Bebbington, 1999) o de promoción } \\
\text { (Foust, 2009). }\end{array}$ & $\begin{array}{l}\text { En ausencia de mecanismos formales } \\
\text { de protección social, las personas } \\
\text { pueden utilizar los distintos tipos de } \\
\text { capital social para protegerse, disminuir } \\
\text { su vulnerabilidad, resolver disputas y } \\
\text { tomar ventajas de nuevas oportunidades } \\
\text { (Woolcock y Narayan, 2000). }\end{array}$ \\
\hline Físico & $\begin{array}{l}\text { Contempla las inversiones físicas en la vivienda, } \\
\text { tierras, así como la infraestructura física y } \\
\text { equipamiento en salud, educación, transporte, } \\
\text { comunicación, agua potable y electrificación, entre } \\
\text { otros. }\end{array}$ & $\begin{array}{l}\text { En función de la resistencia y resiliencia } \\
\text { de los activos físicos a los impactos de } \\
\text { las amenazas. Dependerá de la calidad } \\
\text { de estructuras físicas y su localización } \\
\text { (adaptado de Wilches-Chaux, 1993). }\end{array}$ \\
\hline Cultural & $\begin{array}{l}\text { Son las capacidades, habilidades y saberes } \\
\text { más observables en la vida cotidiana del grupo } \\
\text { social o comunidad. Las estrategias adaptativas } \\
\text { construidas cultural y socialmente también forman } \\
\text { parte (García Acosta, 2009). }\end{array}$ & $\begin{array}{l}\text { La percepción del riesgo, culturalmente } \\
\text { construida, puede producir y reproducir } \\
\text { las condiciones de vulnerabilidad } \\
\text { (García Acosta, 2005). }\end{array}$ \\
\hline Político & $\begin{array}{l}\text { Son las capacidades y habilidades para influir y } \\
\text { vincularse con esferas de poder de mayordisposición } \\
\text { de recursos, sean asociaciones, organizaciones, } \\
\text { instituciones u organismos de carácter público. } \\
\text { Permite la flexibilización de las oportunidades } \\
\text { de acceso e intercambio a diferentes capitales: } \\
\text { humano, financiero o social, pero sin descuidar el } \\
\text { papel que juegan las relaciones de poder (De Haan } \\
\text { y Zoomers, 2005). }\end{array}$ & $\begin{array}{l}\text { Constituye el valor recíproco del nivel } \\
\text { de autonomía que posee un individuo, } \\
\text { grupo o comunidad para la toma de las } \\
\text { decisiones que le afectan, y se encuentra } \\
\text { intimamente ligado a las relaciones } \\
\text { sociales (adaptado de Wilches-Chaux, } \\
\text { 1993). }\end{array}$ \\
\hline Financiero & $\begin{array}{l}\text { Se refiere a los recursos financieros disponibles } \\
\text { para invertir en la construcción de capacidades, vía } \\
\text { créditos o financiamiento para el establecimiento o } \\
\text { desarrollo de empresas o proyectos productivos en } \\
\text { la localidad (Adaptado de Flora et al., 2004). }\end{array}$ & $\begin{array}{l}\text { La disponibilidad de recursos } \\
\text { financieros o de crédito puede disminuir } \\
\text { la vulnerabilidad económica de las } \\
\text { personas, grupos o comunidades. }\end{array}$ \\
\hline Natural & $\begin{array}{l}\text { Es la base de los recursos naturales que } \\
\text { sustentan los medios de vida de los pobladores. } \\
\text { Existen muchos tipos de usuarios y regímenes de } \\
\text { propiedad para los recursos naturales, por lo que } \\
\text { su uso y conservación se encuentra íntimamente } \\
\text { relacionado con la acción colectiva y el capital social } \\
\text { de la comunidad, de cómo utilizan los recursos } \\
\text { naturales para sí mismos y para el bien colectivo } \\
\text { (Adger, 2003). }\end{array}$ & $\begin{array}{l}\text { Las comunidades, en su interacción } \\
\text { con los recursos naturales y su medio } \\
\text { ambiente, construyen nuevos riesgos } \\
\text { y generan nuevas vulnerabilidades } \\
\text { (adaptado a partir de García Acosta, } \\
\text { 2005). }\end{array}$ \\
\hline
\end{tabular}

Fuente: elaboración propia. 
Tabla 2. Tamaño de la muestra

\begin{tabular}{|c|c|}
\hline$n=\frac{N * Z \alpha^{2} p * q}{d^{2} *(N-1)+Z \alpha^{2} * p * q}$ & $\begin{array}{l}\text { En donde: } \\
N=\text { Total de la población }=903 \text { hogares, } \\
Z \alpha=0.05=1.96 \text { al cuadrado (la seguridad es del } 95 \% \text { ), } \\
p=\text { proporción esperada (en este caso, } 93.9 \% \text { de población que } \\
\text { presenta algún grado de vulnerabilidad por carencia social o } \\
\text { pobreza }=0.939 \text { ), } \\
q=1-p \text { (en este caso } 1-0.939=0.061,6.1 \% \text { de población no } \\
\text { pobre y no vulnerable), } \\
d=\text { precisión o error admitido (se calculó la muestra considerando } \\
\text { un } 5 \% \text { de error), } \\
\text { Siendo } n=81 \text { hogares. }\end{array}$ \\
\hline
\end{tabular}

Fuente: elaboración propia.

Tabla 3. Crisis, pérdidas de activos y estrategias familiares ante los diferentes tipos de riesgos

\begin{tabular}{|c|c|c|}
\hline Crisis/riesgo & Pérdida de activos & Estrategias \\
\hline Huracán & $\begin{array}{l}\text { Daños o pérdidas de la vivienda, } \\
\text { pérdida de cosechas, } \\
\text { ingresos, } \\
\text { terrenos, } \\
\text { ganado, } \\
\text { insumos. }\end{array}$ & $\begin{array}{l}\text { Utilizaron sus ahorros, } \\
\text { préstamos y empeños (para comprar semilla), } \\
\text { buscaron apoyo económico del gobierno, extranjeros } \\
\text { y ONG, } \\
\text { empleo temporal (para él y para ella), } \\
\text { pidieron ayuda económica o apoyo a familiares y } \\
\text { amigos, } \\
\text { vendieron activos (terreno y ganado). }\end{array}$ \\
\hline $\begin{array}{l}\text { Desastre por amenaza } \\
\text { natural como } \\
\text { inundación por lluvias } \\
\text { o sequía }\end{array}$ & $\begin{array}{l}\text { Pérdida de cosechas, } \\
\text { daños o pérdidas de la vivienda, } \\
\text { ingresos, } \\
\text { pérdida de negocio familiar. }\end{array}$ & $\begin{array}{l}\text { Utilizaron sus ahorros, } \\
\text { préstamos y empeños, } \\
\text { buscaron ayuda económica del gobierno, } \\
\text { vendieron algún activo (alhajas y ganado), } \\
\text { redujeron los gastos del hogar. }\end{array}$ \\
\hline $\begin{array}{l}\text { Pérdida de empleo o } \\
\text { quiebra del negocio } \\
\text { de algún miembro del } \\
\text { hogar }\end{array}$ & $\begin{array}{l}\text { Ingresos, } \\
\text { pérdida de producción o } \\
\text { cosecha, } \\
\text { terreno, } \\
\text { automóvil, } \\
\text { ganado. }\end{array}$ & $\begin{array}{l}\text { Buscaron otro empleo o pusieron su propio negocio, } \\
\text { vendieron algún activo, } \\
\text { pidieron ayuda económica a familiares y amigos, } \\
\text { pidieron apoyo del gobierno (presidencia municipal, } \\
\text { principalmente). }\end{array}$ \\
\hline $\begin{array}{l}\text { Enfermedad de algún } \\
\text { miembro del hogar o } \\
\text { familiar cercano }\end{array}$ & $\begin{array}{l}\text { Ingresos, } \\
\text { ganado, } \\
\text { terreno. }\end{array}$ & $\begin{array}{l}\text { Utilizaron todos sus ahorros, } \\
\text { préstamos y empeños, } \\
\text { vendieron algún activo (terreno, automóvil, ganado, } \\
\text { alhajas), } \\
\text { incapacidad por el trabajo, } \\
\text { buscaron apoyo económico del gobierno (municipal), } \\
\text { redujeron los gastos del hogar, } \\
\text { pidieron ayuda económica a familiares. }\end{array}$ \\
\hline $\begin{array}{l}\text { Abandono o divorcio } \\
\text { del cónyuge }\end{array}$ & $\begin{array}{l}\text { Ingresos, } \\
\text { vivienda, } \\
\text { terreno. }\end{array}$ & $\begin{array}{l}\text { Algunos miembros del hogar abandonaron la escuela } \\
\text { para buscar trabajo, } \\
\text { buscaron apoyo de la familia, } \\
\text { apoyos de la presidencia municipal y del DIF. }\end{array}$ \\
\hline
\end{tabular}

Fuente: elaboración propia. 